\section{Translational techniques from cleft to cosmetic rhinoplasty}

\author{
Helen L Stark ${ }^{1}$, Charles A Fries ${ }^{2}$, Nigel S Mercer \\ ${ }^{1}$ Nuffield Department of Surgical Sciences, University of Oxford, Oxford; ${ }^{2}$ Department \\ of Plastic Surgery, Oxford University Hospitals NHS Foundation Trust, Oxford; ${ }^{3}$ The \\ Cleft Unit of the South West of England, Bristol Dental Hospital, Bristol, UK
}

Correspondence: Helen L Stark

Nuffield Department of Surgical Sciences, University of Oxford, John Radcliffe Infirmary, Headley Way, Oxford OX3 9DU, UK

Tel: +44-1865-231405, E-mail helen.stark@gmail.com

Received: February 21, 2018 • Revised: May 9, 2019 • Accepted: June 21, 2019 pISSN: 2234-6163 • elSSN: 2234-6171

https://doi.org/10.5999/aps.2018.00185・Arch Plast Surg 2020;47:110-113

Copyright (C) 2020 The Korean Society of Plastic and Reconstructive Surgeons

This is an Open Access article distributed under the terms of the Creative Commons Attribution

Non-Commercial License (https://creativecommons.org/licenses/by-nc/4.0/) which permits

unrestricted non-commercial use, distribution, and reproduction in any medium, provided the original

work is properly cited.

\section{Introduction}

Rhinoplasty is considered a highly complex operation in both cosmetic and reconstructive practice. However, despite its potential pitfalls, a high degree of patient satisfaction can be achieved. Trends in European and North American cosmetic practice have shifted from patients seeking a "supra-normal" appearance to a "normal" appearance. As such, they seek a more individualized result, and the avoidance of an "operated-upon" look [1,2]. More so than ever therefore, rhinoplasty surgeons must have a broad armamentarium of skills and techniques at their disposal to achieve optimal outcomes and patient satisfaction.

The cleft nose deformity is characteristic, but each is also unique. Thus, the skills developed by cleft surgeons in recognizing particular aspects of deformities and attaining individualized outcomes are closely aligned to this contemporary paradigm [3]. During training, plastic surgeons first encounter rhinoplasty in the fields of reconstructive, post-traumatic or cleft surgery. Addressing the anatomic anomalies present facilitates a better understanding of the anatomic and physiologic concepts that are translated to cosmetic practice. It is therefore instructive to reflect on the translatable lessons that may be gleaned from the practice of cleft rhinoplasty surgery.

In this paper, we describe the aberrant anatomy of the cleft nose and illustrate the conceptual approach to its correction. We also discuss a practical approach to performing rhinoplasty surgery.

\section{Anatomy}

The basis for correcting any deformity is an understanding of the "normal" state of affairs and how it has been altered by pathology and competing, unopposed forces. In cleft and cosmetic rhinoplasty patients, it is important to consider both the skeletal and soft tissue anatomy and the forces that are at play during any corrective procedure. If the nasal skeleton is likened to an interdependent tripod with the septum as the central support and the nasal walls and lower lateral cartilages as lateral support, it is possible to conceptualize how an imbalance of forces or a deficiency in one area leads to deformity [4].

Cleft nasal deformity is one of the most severe nasal abnormalities seen in regular practice. The caudal septum is deviated to the noncleft side, whilst the posterior septum is convex on the cleft side. The cartilaginous septum buckles at the junction with the vomer, usually at $90^{\circ}$. The columella usually appears shortened and displaced towards the side of the cleft because the medial crura and domes are pulled apart by the unbalanced forces of the mimetic muscles. There is poor tip projection. The lower lateral cartilage on the cleft side is flattened and displaced inferolaterally as a result, giving a slumped appearance and contributing to the lack of tip projection. In addition, the effects of growth lead to further changes [3].

\section{How this applies to cosmetic rhinoplasty}

Many deformities may prompt a patient to request cosmetic rhinoplasty surgery. Patients may present with dissatisfaction regarding the shape and/or size of their nose, and the anatomic basis for this dissatisfaction is myriad in its complexity. During the consultation, the surgeon must establish the specific concerns of the patient in detail, and which manoeuvres relating to the underlying structures may be required. Techniques derived from cleft practice regarding the manipulation of the size, shape and position of the septum and lower lateral cartilages can address many of these concerns. Hence, understanding the comparison between normal and cleft anatomy helps the surgeon diagnose and treat deformities of the nose in general.

\section{Surgical technique-concepts}

We believe that the central tenet of rhinoplasty, cleft or cosmetic, is correction of the nasal septum. The septum provides a stable framework to build upon when adjusting other nasal abnormalities, as by correction of the central strut of the "tripod," the remaining abnormal structures are drawn into position. An overriding principle of this paper is Newton's third law, which states that "for every action there is an equal and opposite reaction." When using grafts to support the septum, we recommend using grafts on both sides of the septum. These are commonly referred to as "strut" grafts, but rhinoplasty surgeons should view any structural graft as an engineer does a girder. Just as with a girder, if cartilage grafts are just placed in space, they will be ineffective because opposing forces will push them back. Similarly, attention to the nasal tip requires correct fixation of structures rather than simple placement of grafts, ensuring that the supporting skeleton will not angulate, rotate or deviate by fixing the structures according to the principles of structural engineering. 


\section{Surgical technique-practical steps}

\section{History}

A comprehensive history must be taken to establish the patient's fitness to undergo surgery, any pathological conditions relating to the nose or nasal airway, and a detailed exploration of the patient's specific cosmetic concerns, expectations, and psychological suitability to undergo cosmetic surgery. This is the foundation of developing an operative plan with the patient.

\section{Examination}

The nose must be examined systematically, in the context of the rest of the face. Various proportions may be used when analyzing the nose preoperatively, and systems such as that described by Rohrich and Ahmad [5] may be employed. In this manner, each component of the nose is evaluated for any abnormalities that may contribute to the perceived deformity.

Clinical photography is mandatory, to document findings, to aid in communication, to complete the medical record and for medicolegal purposes. The use of drawings or software to manipulate these images electronically is also helpful (Fig. 1). Understanding how the patient views his or her nose will help the surgeon decide on an appropriate preoperative plan.

\section{Consent}

Consent should be at least a two-stage process. Having discussed the proposed procedure, the patient should then have a cooling-off period before returning to confirm what they would like to achieve.

\section{Surgical technique}

Patients are anaesthetized using total intravenous anesthesia, intranasal cocaine, and $2 \%$ lidocaine with 1:80,000 adrenaline. One dose of co-amoxiclav $(1.2 \mathrm{~g})$ and tranexamic acid $(1 \mathrm{~g})$ is administered intra-

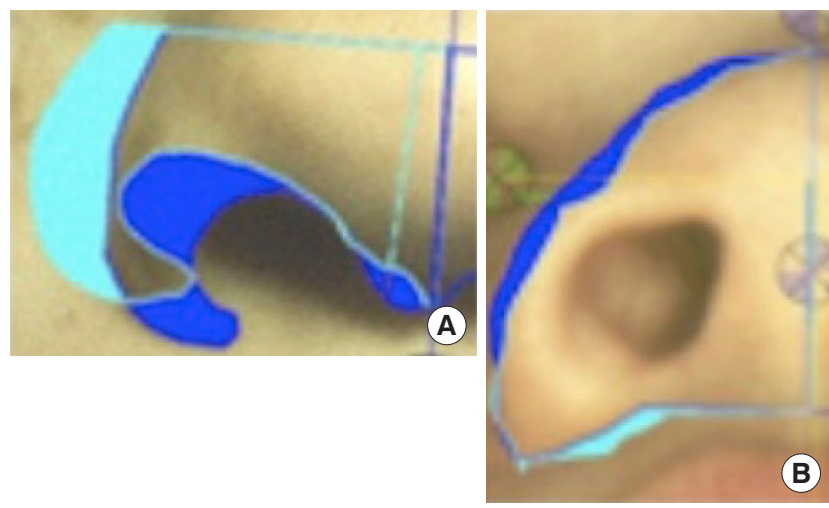

Fig. 1.

(A, B) Nasal views (left front and right basal) analyzed with Symnose. Symnose is a computer program that assesses the symmetry of noses, nostrils and lips by overlying the left onto the right side across a defined meridian. The blue color highlights areas of asymmetry between the two sides. Cyan is used to denote the left side and dark blue is used on the right. venously at induction. Intravenous paracetamol $(1 \mathrm{~g})$ is delivered intraoperatively. Dexamethasone is also used to reduce swelling and as an anti-emetic. An open approach is used in almost all cases, save those requiring very little manipulation of the tip structures. A "V" incision on the columella is the senior author's preferred access incision (Fig. 2).

The critical part of a cleft rhinoplasty repair is addressing the septal deformity. As mentioned previously, the septum is the keystone of support for the rest of the nasal structures, and in order to achieve a satisfactory outcome it must be accurately positioned. This often requires the use of grafts to provide structural support to maintain the new position, as predicted by Newton's third law. When performing septal work or harvesting a graft, it is essential to avoid septal perforation.

Firstly, a plan should be made regarding which grafts will be required, and then the surgeon dissects and harvests them from the septum, ensuring that the septum is left as an "L" strut with a width of $7-10 \mathrm{~mm}$ in the upper and lower dimensions. We recommend the use of strut grafts applied as "girders" on either side to straighten the septum and maintain it in position. Straight grafts are placed on both sides of the septum and sutured across with horizontal mattress sutures. The septum is incised or excised to achieve this straightening. This provides the necessary "equal and opposite" force to hold the septum in its desired new position (Fig. 3). It is crucial to remove the

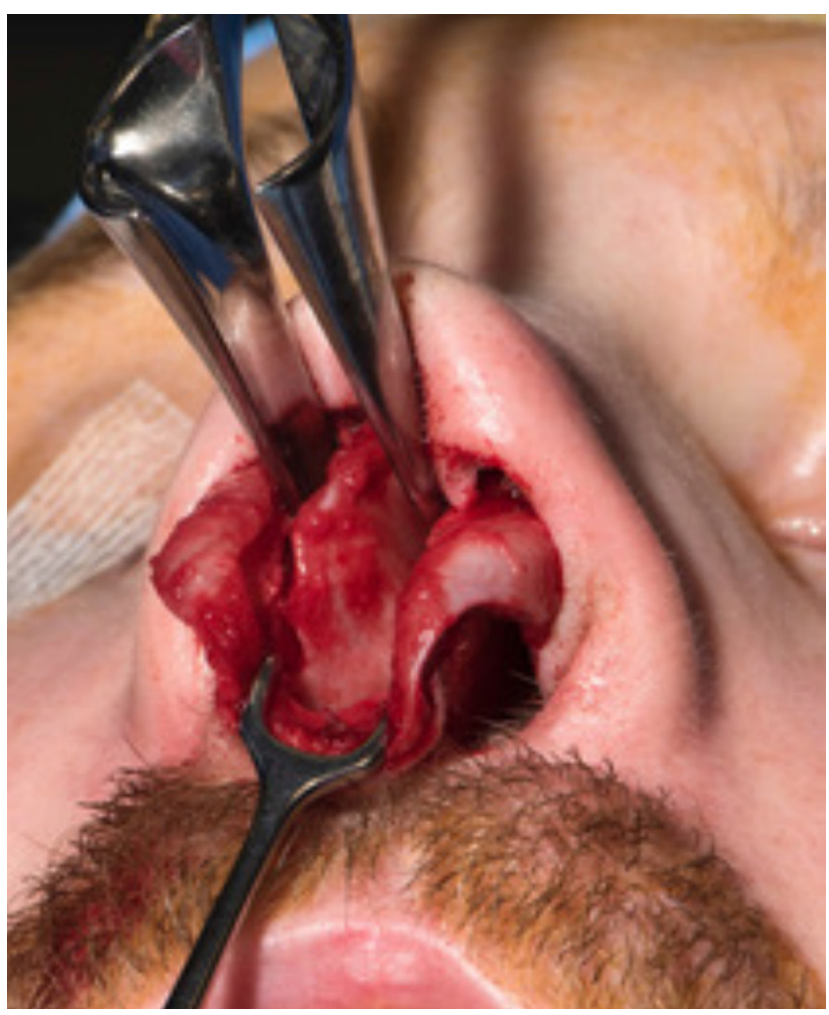

Fig. 2.

Open approach to rhinoplasty, demonstrating a clear view of the septum. 


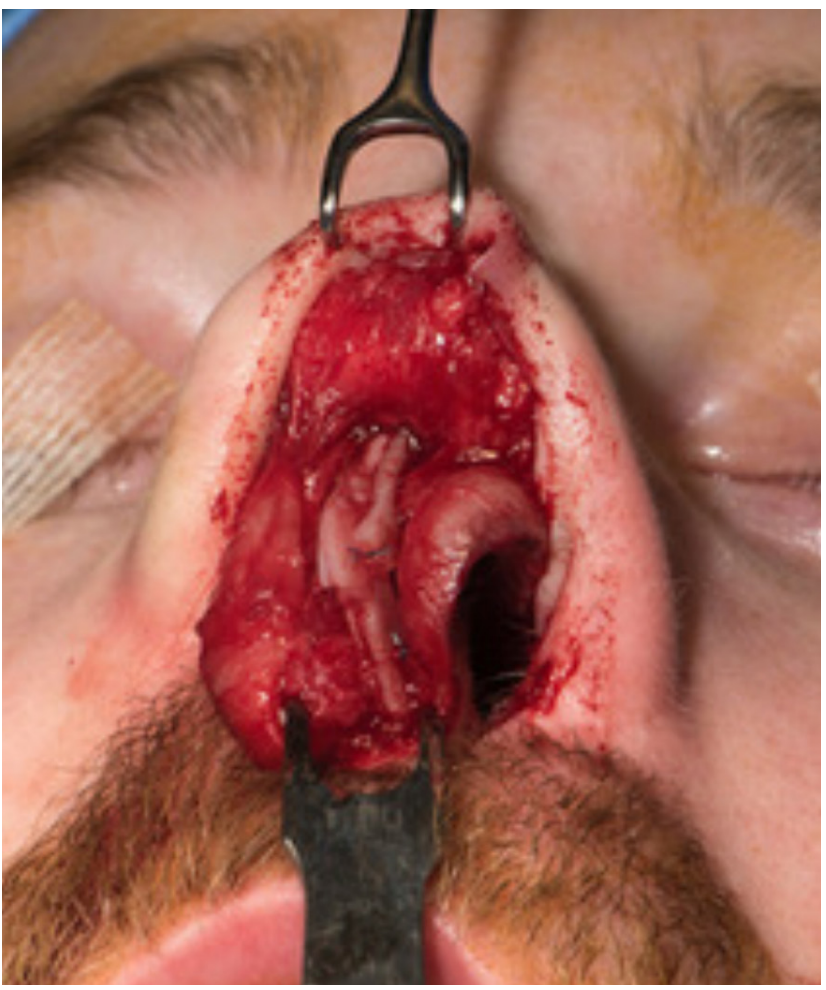

Fig. 3.

Addressing the septal deformity, note the strut (girder) graft in position on either side of the septum, held in place with a horizontal mattress suture.

bent cleft septum from the nasal spine to allow for this straightening. It is advisable to fix the septum to the maxilla in the dental midline, but the surgeon must be aware that the dental midline may not be in line with the anterior nasal spine due to previous orthodontic treatment.

Once the septum has been satisfactorily repositioned and the nasal dorsum corrected, osteotomies may be required to close an open roof deformity or to narrow the nasal dorsum. We prefer curved, lowto-low, internal osteotomies following the contour of the maxilla. The nasal tip is then addressed (Fig. 4). Tip and dome manipulations are planned to normalize the nose, which may include de-rotation, centralization and equalization of the cartilaginous structures. The trend now is towards minimal reduction by means of a cephalic trim. Finally, the procerus muscle may be removed. A plaster of Paris splint is applied and stays in place for 2 weeks.

\section{Conclusions}

The modern rhinoplasty surgeon, striving to create an aesthetically pleasing "normal"-in distinction from "supranormal"- nose in line with the specific aesthetic goals of their patient, must have a wide armamentarium of techniques and skills. Evaluating the altered anatomy of the cleft nasal deformity, in particular manipulating the septum and lower lateral cartilages whilst respecting Newton's third law, as

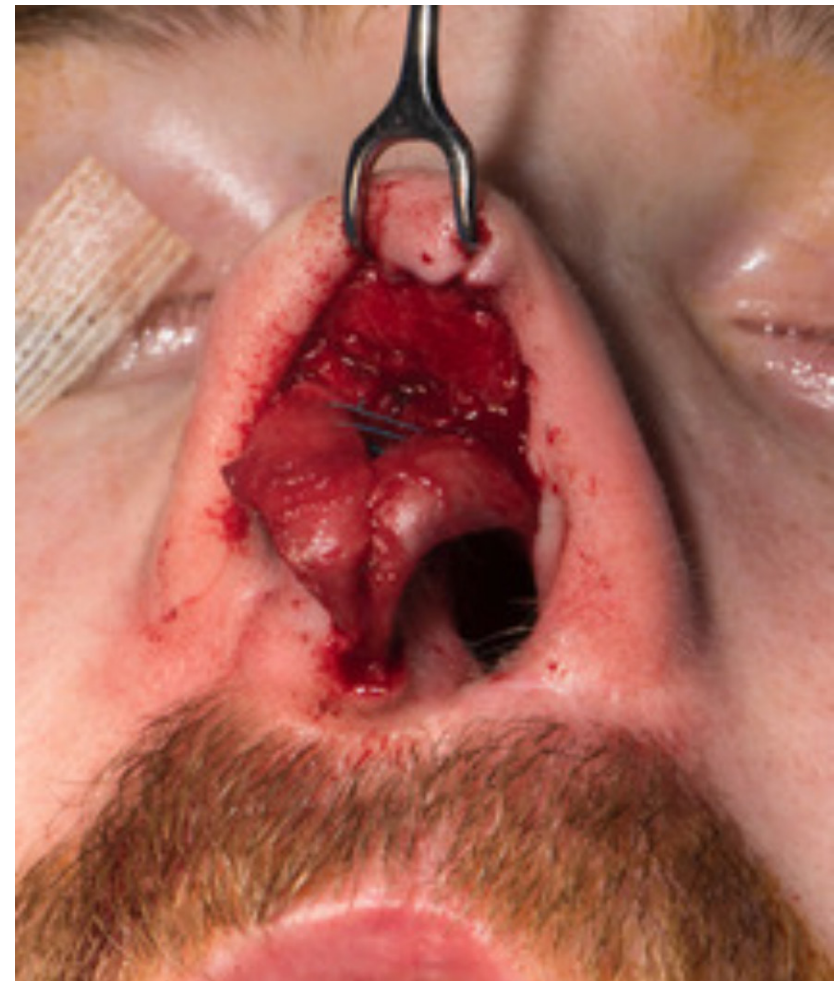

Fig. 4.

Tip reconstruction and adjustment of the position of alar cartilage.

described above, offers rewarding insights and skills that are translatable to the cosmetic arena.

Notes

Conflict of interest

No potential conflict of interest relevant to this article was reported.

\section{Ethical approval}

The study was performed in accordance with the principles of the Declaration of Helsinki. Written informed consent was obtained.

\section{Patient consent}

The patient provided written informed consent for the publication and the use of his images.

Author contribution

Conceptualization: Mercer NS. Data curation: Mercer NS. Formal analysis: Stark HL, Fries CA, Mercer NS. Project administration: Stark HL, Fries CA, Mercer NS. Visualization: Mercer NS. Writing of original paper: Stark HL, Fries CA. Review and editing: Stark HL, Fries CA, Mercer NS. 


\section{ORCID}

Helen L Stark https://orcid.org/0000-0002-9360-9864

Charles A Fries https://orcid.org/0000-0003-0128-5688

Nigel S Mercer https://orcid.org/0000-0002-7259-5088

\section{References}

1. Wallop H. 100 years of plastic surgery. The Telegraph. 2015 Jul 10. Available from: https://www.telegraph.co.uk/women/womenshealth/11731223/100-years-of-plastic-surgery.html.
2. Janin J, Ahmad J, Rohrich R. Rhinoplasty. In: Thorne CH, Chung KC, Gurtner GC, et al. editors. Grabb and Smith's plastic surgery. New York: Lippincott Williams \& Wilkins; 2014. p. 512-29.

3. Van Beek AL, Hatfield AS, Schnepf E. Cleft rhinoplasty. Plast Reconstr Surg 2004;114:57e-69e.

4. Stal S, Hollier L. Correction of secondary deformities of the cleft lip nose. Plast Reconstr Surg 2002; 109:1386-92.

5. Rohrich RJ, Ahmad J. Rhinoplasty. Plast Reconstr Surg 2011;128:49e$73 \mathrm{e}$. 